\title{
Capacidad de nado del pingüino fósil Inkayacu paracasensis Clarke, 2010 (Aves: Spheniscidae) con la tasa metabólica basal o estándar
}

\author{
Iván MEZA-VÉLEZ
}

Departamento de Paleontología de Vertebrados, Museo de Historia Natural, Universidad Nacional Mayor de San Marcos. Lima 11, Perú; imv2999@gmail.com

*Corresponding author

Meza-Vélez, I. 2020. Capacidad de nado del pingüino fósil Inkayacu paracasensis Clarke, 2010 (Aves: Spheniscidae) con la tasa metabólica basal o estándar. [Swimming capacity of the fossil penguin Inkayacu paracasensis Clarke, 2010 (Aves: Spheniscidae) with the basal or standard metabolic rate]. Spanish Journal of Palaeontology, 35 (2), 185-196.

Manuscript received 20 August 2019

Manuscript accepted 2 June 2020 https://doi.org/10.7203/sjp.35.2.18482

(C) Sociedad Española de Paleontología ISSN 2255-0550

\section{RESUMEN}

El objetivo del estudio es calcular la capacidad de nado del pingüino extinto Inkayacu paracasensis en términos de la velocidad máxima de natación estimada, utilizando un método teórico biomecánico (mecánica de fluidos) aplicado a reptiles marinos mesozoicos y cetáceos vivos. A efectos comparativos, este parámetro también se calcula para el pingüino africano de patas negras Spheniscus demersus y el pingüino Emperador Aptenodytes forsteri. Los resultados muestran una velocidad máxima estimada de $2,8-3,4 \mathrm{~m} / \mathrm{seg}, 2,8-3,5 \mathrm{~m} / \mathrm{seg}$ y $3,0-3,7$ $\mathrm{m} / \mathrm{seg}$, respectivamente. Las velocidades máximas observadas registradas en estudios con especies en cautiverio y en la naturaleza permiten corroborar los valores calculados para las especies vivas, con un error máximo de alrededor del $10 \%$ en el caso de A. forsteri. Por lo tanto, el pingüino Emperador e Inkayacu han desarrollado velocidades similares de natación. Además, se sugiere que la frecuencia del batido de la aleta, longitud de la zancada y fases de deslizamiento habrían sido similares en ambas especie de cuerpo grande, en contraste con las de menor tamaño. También se analiza la influencia del modelo de flujo (número de Reynold) en las tres especies de pingüinos. Se sugiere que la morfología del cráneo de Inkayacu podría ser una ventaja en la locomoción con respecto al pingüino Emperador.

Palabras clave: Inkayacu paracasensis, Aptenodytes forsteri, velocidad máxima, número de Reynolds, biomecánica.

\begin{abstract}
The aim of the study is to calculate the swimming capability of the extinct penguin Inkayacu paracasensis in terms of estimated maximum swimming speed, based on a theoretical biomechanical (fluid mechanics) method, which was previously applied to Mesozoic marine reptiles and living cetaceans. For comparison purposes, this parameter is also calculated for the African black-footed penguin Spheniscus demersus and the Emperor penguin Aptenodytes forsteri. Results show an estimated maximum speed of $2.8-3.4 \mathrm{~m} / \mathrm{s}$, $2.8-3.5 \mathrm{~m} / \mathrm{s}$ and $3.0-3.7 \mathrm{~m} / \mathrm{s}$ for the three species respectively. Studies with species in captivity and in the wild allows corroborating the estimated values for the living species, with a maximum error of about $10 \%$ in the case of $A$. forsteri. Therefore, the Emperor penguin and Inkayacu have developed similar swimming velocities. It is suggested that wing beat frequency, stride length and gliding phases are similar in both large bodied species, in contrast of the small ones. In addition, it is also analyzed the influence of the flow model (Reynold's number) in the three penguin species. It is suggested that skull morphology of Inkayacu could be an advantage in locomotion with respect of the Emperor penguin.
\end{abstract}

Keywords: Inkayacu paracasensis, Aptenodytes forsteri, maximum velocity, Reynold's number, biomechanics. 


\section{INTRODUCCIÓN}

Reconstruir la naturaleza que existía en tiempos prehistóricos es una tarea difícil, pues los restos que ha dejado el paso del tiempo son escasos y muchas veces poco conservados, más aun en aquellos especímenes que difieren significativamente de la morfología de sus descendientes actuales o que carecen totalmente de parientes modernos (Shockey et al., 2007). La paleontología es la ciencia que se enfrenta día a día con ese problema; por ejemplo, no se cuenta con un gran número de taxa o con los órganos internos, músculos y tejidos de los fósiles más antiguos. Por esta razón, el trabajo del paleontólogo se ve enriquecido al utilizar otras especialidades que puedan arrojar luces sobre cómo habrían sido, vivido o desplazado los animales extintos. De aquellas ciencias, la biomecánica es la encargada de reconstruir la locomoción de los vertebrados fósiles.

La mecánica es la rama de la física que trata sobre el movimiento y las fuerzas implicadas en él, mientras que la biomecánica ha sido definida como el estudio del movimiento de los entes vivos usando la ciencia de la mecánica (Hatze, 1974). Si se quiere un estudio más especializado, como el nado de un plesiosaurio o el vuelo de las aves, la biomecánica tomará elementos de la mecánica de fluidos que estudia las leyes del movimiento de los líquidos y de los gases y su interacción con los sólidos (Yavorski, 1977). Así, la biología y la paleontología pueden estudiar y reconstruir, desde la perspectiva de la física, las adaptaciones y diseños de los organismos, y cuantificar sus capacidades de locomoción frente al medio fluido donde habitan (o habitaban).

Identificamos que el problema es la carencia de información en el estudio de la locomoción de los vertebrados fósiles, esto se ve reflejado de manera concreta en el caso de Inkayacu paracasensis Clarke, 2010. El pingüino gigante $I$. paracasensis pertenece al Eoceno superior (aproximadamente 36 millones de años) de la localidad de Yumaque, Reserva de Paracas, Perú (Clarke et al., 2010), cuyo holotipo está casi completo y se guarda en el Departamento de Paleontología de Vertebrados del Museo de Historia Natural de la UNMSM.

El desarrollo de la biomecánica de vertebrados fósiles es de gran importancia, debido a que la reconstrucción de la locomoción amplía o corrige la información ecológica o de comportamiento de aquellos vertebrados del pasado. Para los fines de la paleontología un aporte interdisciplinario que ayude a completar el rompecabezas de una naturaleza que ya no existe, está plenamente justificado.

El objetivo de esta investigación es evaluar la capacidad de nado de Inkayacu paracasensis en términos de velocidad estimada, tal como ha realizado Massare (1988) con reptiles marinos fósiles y cetáceos actuales. En este sentido, este trabajo es pionero en aplicar dicha metodología a aves acuáticas, extintas y actuales. Los resultados se basan en la tasa metabólica basal o estándar, aunque se puede usar para diferentes condiciones fisiológicas de una especie dada (Eckert et al., 1994), no es una tasa metabólica activa. Sin embargo, se justifica el uso del modelo en pingüinos por dos razones: 1) la aproximación de la forma del cuerpo fusiforme del ave acuática a través del elipsoide prolato del modelo es igual o más realista que en algunos grupos de reptiles marinos del Mesozoico estudiados en el trabajo de Massare (1988); 2) los valores calculados de velocidad de cetáceos actuales no difieren significativamente de los observados, por lo que validan el modelo.

Los pingüinos (familia Spheniscidae) son un grupo distintivo de aves no voladoras, marinas pelágicas, ampliamente distribuidas en las aguas frías del hemisferio sur (Williams, 1995). La familia comprende 18 especies recientes y numerosas especies fósiles (Simpson, 1946; Stonehouse, 1975). Probablemente sea el ave acuática que muestre la más extensa serie de especializaciones morfológicas para el nado (Clark \& Bemis, 1979), para otros autores es el grupo más altamente especializado para la locomoción submarina (Raikow, 1985).

Estas características han convertido a los pingüinos en objeto de numerosos estudios. Así tenemos investigaciones sobre su enigmática filogenia (Simpson, 1946; Slack et al., 2006), la cinemática del nado (Clark \& Bemis, 1979), patrones morfométricos (Livezey, 1989), metabolismo del nado (Luna-Jorquera \& Culik, 2000), estructura del esqueleto (Zusi, 1975), etc., en las especies recientes. Sin embargo, los trabajos sobre locomoción en aves acuáticas y, más específicamente en pingüinos fósiles, son muy escasos, refiriéndose exclusivamente a estimaciones del tamaño y la masa del cuerpo, estudios morfométricos, sistemática y filogenia.

En cuanto a la locomoción de animales extintos nadadores, Massare (1988) ha estudiado las propiedades hidrodinámicas de las diferentes formas del cuerpo y modos de propulsión de reptiles marinos del Mesozoico, como Plesiosaurus, Mosasaurus o Ichthyosaurus. También lo ha hecho con cetáceos actuales, para verificar el método obteniendo estimados de velocidad, que son comparados con las velocidades observadas. Sin embargo, el modelo tiene limitaciones; por ejemplo, en el caso de la velocidad del nado de los cetáceos modernos las limitaciones del modelo son las siguientes: 1) la eficiencia muscular se define en 0,2 , para cetáceos y todos los grupos; 2) la eficiencia propulsora o eficiencia hidrodinámica se define en 0,85 , medido en el nado oscilatorio axial del delfín nariz de botella, cifra aplicada a todos los cetáceos, donde el movimiento muscular se fundamenta en la aleta caudal. El valor y el impacto son diferentes en reptiles, que poseen movimiento lateral. En pingüinos, la eficiencia propulsora está sustentada, principalmente, por los movimientos del ala, los cuales conservan los grados de libertad; 3) la constante metabólica promedio en mamíferos se define en 415 , y es aplicado a los cetáceos. Sin embargo, las tasas metabólicas en las aves son más altas que las reportadas 
en reptiles o en mamíferos; 4) se utiliza la tasa metabólica basal o estándar, determinada en estado de ayuno y reposo.

\section{Abreviaciones institucionales}

UNMSM, Universidad Nacional Mayor de San Marcos; MUSM, Museo de Historia Natural de la Universidad Nacional Mayor de San Marcos, Lima, Perú.

\section{MATERIAL Y MÉTODOS}

\subsection{Material biológico}

Holotipo de Inkayacu paracasensis MUSM 1444 de la colección del Departamento de Paleontología de Vertebrados del Museo de Historia Natural de la Universidad Nacional Mayor de San Marcos (Lima, Perú). El fósil casi completo representa a un pingüino con una longitud estimada de $1,5 \mathrm{~m}$ y una masa conservadoramente estimada de 54,6 a $59,7 \mathrm{~kg}$ (Clarke et al., 2010), aproximadamente el doble del promedio de masa del pingüino Emperador (Stonehouse, 1967). Se usará la descripción de Inkayacu paracasensis realizada por Clarke et al. (2010).

\subsection{Métodos}

El método seguido es una aproximación al modelo de Massare (1988) para determinar las variaciones dinámicas del movimiento de Inkayacu paracasensis en relación a pingüinos actuales (Aptenodytes forsteri Gray, 1844 (pingüino Emperador) y Spheniscus demersus Linnaeus, 1758) con el fin de comparar los resultados de las tres especies con los valores observados de las especies actuales.

Los pasos son los siguientes:

a) Se calcula la relación longitud-diámetro del cuerpo, valor determinante para la resistencia del agua. Se utiliza:

$$
\mathrm{F}=\mathrm{L} / \mathrm{W}
$$

Donde F: "fíneness ratio" (razón de esbeltez), L: longitud del cuerpo, W: diámetro medio del cuerpo (Bainbridge, 1961; Alexander, 1968).

b) Se relaciona la potencia total de salida del animal (P) y la resistencia del agua (D) con la siguiente formula:

$$
\mathrm{DU}=\mathrm{eP}
$$

Donde U: velocidad del cuerpo, e: proporción de la potencia total que pone en movimiento al animal (Wu, 1977; Weihs, 1977).
Si P se toma como la tasa metabólica aeróbica máxima, entonces la velocidad será la velocidad de nado sostenida máxima. Así, la resistencia total (D), la proporción de la potencia total de salida utilizada para la locomoción (e), y la potencia de salida aeróbica máxima $(\mathrm{P})$ deben ser estimadas con el fin de calcular una velocidad máxima de natación sostenida (Massare, 1988).

c) Se estima la resistencia que ofrece el agua en las especies seleccionadas, la cual se da en la columna de agua y cuyo diámetro equivale a la sección transversal (área promedio) del cuerpo dada las condiciones de flujo. La resistencia está dada por:

$$
D=\left(\rho U^{2} A C_{d}\right) / 2
$$

D también es denotado como Fr (fuerza de resistencia). En la ecuación, p: densidad del fluido $\left(1.000 \mathrm{~kg} / \mathrm{m}^{3}\right.$ para el agua), U: velocidad del cuerpo con respecto al fluido, A: área de la superficie total del cuerpo, $\mathrm{C}_{\mathrm{d}}$ : coeficiente de arrastre, que depende de la forma del objeto pero también de las condiciones de flujo, es decir, del número de Reynolds (Massare, 1988; Jou et al., 1994).

d) Se calculará el área de la superficie del cuerpo. Para ello se considerará el cuerpo como el volumen de un esferoide prolato. Las formas corporales deben ser aproximadas por alguna forma geométrica (Massare, 1988). Siguiendo el modelo de Massare (1988), se asumirá la sección transversal circular perpendicular a la dirección del movimiento. A diferencia de la mayoría de los peces, los pingüinos tienen secciones transversales casi circulares. La longitud del animal (L) es el eje mayor del elipsoide alargado, la profundidad (ancho) del cuerpo (W) es la longitud del eje menor y por lo tanto el diámetro de la sección transversal circular. Entonces, el área de superficie (A) está dada por:

$$
A=2 \pi\left(\frac{W}{2}\right)^{2}+2 \pi \frac{\left(\frac{L W}{4}\right)}{E} \sin ^{-1} E
$$

Donde E es la excentricidad de la sección transversal elíptica, que está definida como:

$$
E=\frac{\sqrt{\left(\frac{L}{2}\right)^{2}-\left(\frac{W}{2}\right)^{2}}}{\left(\frac{L}{2}\right)}
$$

e) Para estimar la fricción se necesita conocer el coeficiente de arrastre, también depende de la forma del cuerpo que se ha supuesto: un esferoide prolato. La fricción de arrastre es mucho menos cuando el modelo de flujo en contacto con la superficie del cuerpo es laminar que cuando es turbulento. En consecuencia, la resistencia total en un cuerpo se reduce a la transición de un flujo laminar a una capa límite turbulenta (Hoerner, 1965; Alexander, 1968). El coeficiente de resistencia mínima, que se produce en esta 
zona de transición, se puede aproximar por un elipsoide alargado por:

$$
C_{d}=C_{f}\left[1+1.5\left(\frac{W}{L}\right)^{1.5}+7\left(\frac{W}{L}\right)^{3}\right]
$$

Donde $\mathrm{C}_{\mathrm{f}}$ es el componente de fricción de la piel del coeficiente de arrastre, que es de aproximadamente 0,004 (Hoerner, 1965). La velocidad sostenida de nado calculada usando esta expresión para el coeficiente de arrastre sería el límite superior de la velocidad sostenida. La aproximación de esta ecuación se cumple para los cuerpos elipsoidales profundamente sumergidos, donde la profundidad de inmersión es superior a cinco veces el diámetro del cuerpo (Hoerner, 1965). Sin embargo, cerca de la superficie del agua la fricción de onda se vuelve importante. La resistencia o fricción de onda cerca de la superficie es del mismo orden de magnitud que el arrastre debido solo a la forma del cuerpo (Hoerner, 1965). La fricción de onda se calcula duplicando el coeficiente de resistencia:

$$
C_{d} \text { total }=2 C_{f}\left[1+1.5\left(\frac{W}{L}\right)^{1.5}+7\left(\frac{W}{L}\right)^{3}\right]
$$

f) Se estimará la proporción de la potencia de salida usada en la locomoción. Cuando la potencia total de salida (P) se mide como la tasa metabólica, la proporción de energía disponible para la locomoción se puede expresar como (Massare, 1988):

$$
\mathrm{e}=\mathrm{e}_{\mathrm{p}} \mathrm{e}_{\mathrm{m}}
$$

Donde $\mathrm{e}_{\mathrm{p}}$ : propulsión o eficiencia hidrodinámica, que depende del modo de propulsión, en particular para propulsión basada en las aletas pectorales (remo), como en los pingüinos, cuyo valor es de 0,15 a 0,30 (Blake, 1980); $\mathrm{e}_{\mathrm{m}}$ : eficiencia muscular, que es la eficiencia de convertir energía bioquímica en energía mecánica $(\mathrm{Wu}$, 1977; Webb, 1977). Se asumirá $\mathrm{e}_{\mathrm{m}}=0,20$, tal como lo hace Massare (1988) para todos los grupos de animales: cetáceos y reptiles marinos del Mesozoico.

g) La tasa metabólica es una medida de la potencia total de salida $(P)$. Específicamente, $P$ es la tasa metabólica basal (BMR) o la tasa metabólica estándar (SMR) (Eckert et al., 1994). La BMR es la tasa estable del metabolismo energético medida en mamíferos y aves en condiciones de mínimo estrés ambiental y fisiológico, y después de que el ayuno detenga temporalmente los procesos absortivos y digestivos; mientras que la SMR se define como el metabolismo de un animal en reposo y en ayunas a una temperatura corporal dada (Eckert et al., 1994). Según Kleiber (1947) y Wilkie (1977) una buena aproximación de la tasa metabólica de un organismo está dada por:

$$
P=a M^{0,75}
$$

Donde $\mathrm{M}$ es la masa del animal y a es una constante, determinada por el nivel de actividad del animal, si es homeotermo, poikilotermo o unicelular (Massare 1988); a también es definido como un coeficiente de proporcionalidad que varía según la especie (Eckert et al., 1994). El exponente 0,75 ha sido determinado empíricamente, expresa la tasa de cambio de $P$ al variar la masa corporal (Eckert et al., 1994).

Si $P$ es medida como la tasa metabólica aeróbica máxima (Massare, 1988), en julios/seg, entonces la constante metabólica a está en el intervalo de 11,6-29 para los reptiles y 300-530 para los mamíferos y aves (convertido de la energía máxima a través del metabolismo del ejercicio aeróbico en Bakker, 1975; Tabla 21.1).

Para esta investigación, son muy importantes las características del metabolismo que se va a utilizar. La BMR y la SMR se determinan en el animal en un estado controlado y en reposo, y por ello no natural. Estas mediciones son simples líneas de base útiles para comparar entre especies y para diferentes condiciones fisiológicas de una especie dada (Eckert et al., 1994). Si bien es útil usar esta tasa, según Eckert et al. (1994), no es una tasa metabólica activa, que corresponde al nado de un ave acuática. Entonces, esta tasa metabólica basal constituye una limitación del modelo.

h) Cálculo de la velocidad de natación (Massare, 1988): combinando las ecuaciones (3), (7), (8) y (9) con la ecuación (2), obtenemos una expresión para la velocidad máxima sostenida de natación, que está dada por:

$$
U=\left(\frac{2 e_{\rho} e_{m} P}{\rho A C_{d}}\right)^{0,3333}
$$

Esta velocidad es la velocidad sostenida máxima, debido a que utiliza la tasa metabólica aeróbica máxima (P), que a su vez usa la energía máxima de una actividad (locomoción) aeróbica metabólica.

Como ya se vio, $\mathrm{P}, \mathrm{A}, \mathrm{y}_{\mathrm{d}}$ son funciones de la longitud y anchura. Mediante la estimación de las diversas constantes, U se puede calcular para un animal dado si su longitud y anchura (o profundidad) del cuerpo son conocidas.

Este cálculo, sin embargo, se basa en una serie de restricciones importantes (Massare, 1988):

1. La estimación de arrastre asume que el animal es un cuerpo rígido y que la oscilación de las extremidades del cuerpo, o de la cola, no contribuye a la resistencia. Por lo tanto, la velocidad de natación calculada a partir de la ecuación (10) puede esperarse que sea más grande que la real.

2. La forma asumida para los animales nadadores determina la estimación del volumen que se utiliza en el cálculo de la potencia de salida (ecuación 9), teniendo en cuenta que la masa $M$ del animal puede ser expresada como el producto del volumen por la densidad. También determina el área de superficie y 
la expresión para el coeficiente de arrastre utilizado en el cálculo de arrastre (ecuación 3). Por lo tanto, afecta tanto al numerador y al denominador de la ecuación (10), y su efecto exacto es difícil de cuantificar.

3. El supuesto de que la fricción de onda es importante duplicó el tamaño del coeficiente de arrastre (ecuación 7). Si se descuida la fricción de onda, la velocidad calculada utilizando la ecuación (10) se incrementa por un factor de solo 1,25. Por lo tanto, esto sería menos crítico que los otros puntos.

i) Finalmente, se comparará el modelo de flujo de Inkayacu con el del pingüino Emperador y de Spheniscus demersus. Se aplicará el número de Reynolds (Re). Este análisis es un complemento al método aplicado por Massare (1988). El Re está dado por la expresión:

$$
R_{e}=\frac{v_{t} l \rho}{\mu}
$$

Donde $\mu$ es la viscosidad dinámica del agua; $l$ es la longitud del cuerpo del animal; y $v_{t}$ es la velocidad del animal.

\section{RESULTADOS}

\subsection{Cálculo de W (ancho del cuerpo)}

Inkayacu paracasensis: Utilizando una foto con tres individuos (Fig. 1) se halló la razón promedio de esbeltez (ecuación 1) del pingüino Emperador, $\mathrm{F}=2,57$.

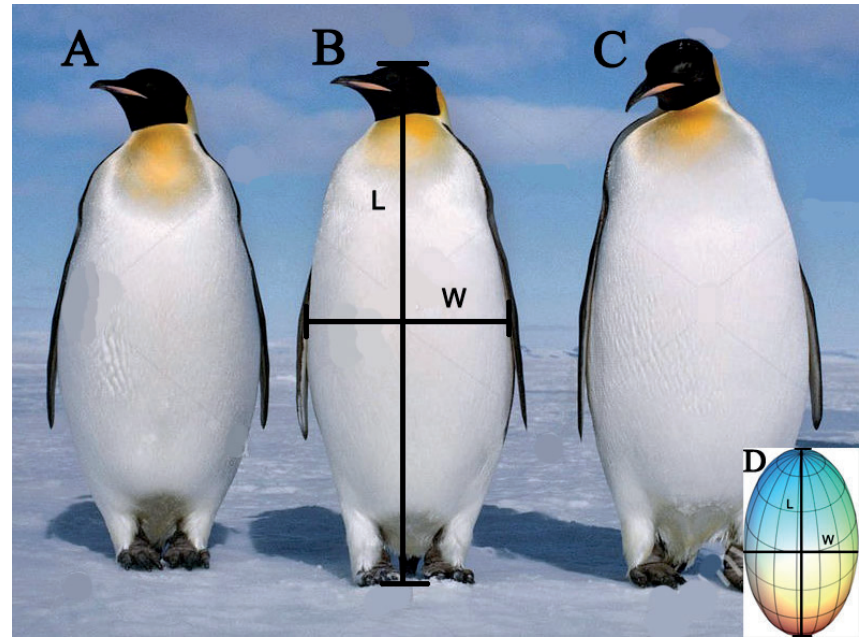

Figura 1. (a-c) Tres individuos de Aptenodytes forsteri. d) Esferoide prolato. L: longitud del cuerpo; W: diámetro medio del cuerpo. Modificado de fotografía: https://www.google.com.pe/search?hl=en-PE\&biw= $1366 \&$ bih $=657 \&$ tbm $=$ isch \&sa $=1 \&$ ei $=$ KlhXXYOUN 8yHggfihqDACA\&q=pinguino + emperador + alamy $+\mathrm{s}$ tock + photo\&oq $=$ pinguino + emperador + alamy + stock+ photo\&gs_l=img.3...
Asumiendo que la forma de Inkayacu paracasensis es similar a la de Aptenodytes forsteri (pingüino Emperador), entonces de $\mathrm{F}=\mathrm{L} / \mathrm{W}$ y para $\mathrm{L}=150 \mathrm{~cm}$ se tiene que $\mathrm{W}$ $=58,4 \mathrm{~cm}(\sim 58)$.

Aptenodytes forsteri: Utilizando $\mathrm{F}=2,57$ y $\mathrm{L}=115$ cm (Stonehouse, 1967), obtenemos $\mathrm{W}=44,7 \mathrm{~cm}(\sim 45)$.

Spheniscus demersus: Utilizando una foto (Fig. 2) se halló la razón de esbeltez de un individuo de $S$. demersus, $\mathrm{F}=2,7$. De F y $\mathrm{L}=70 \mathrm{~cm}$ (Stonehouse, 1967), obtenemos $\mathrm{W}=25,9 \mathrm{~cm}(\sim 26)$

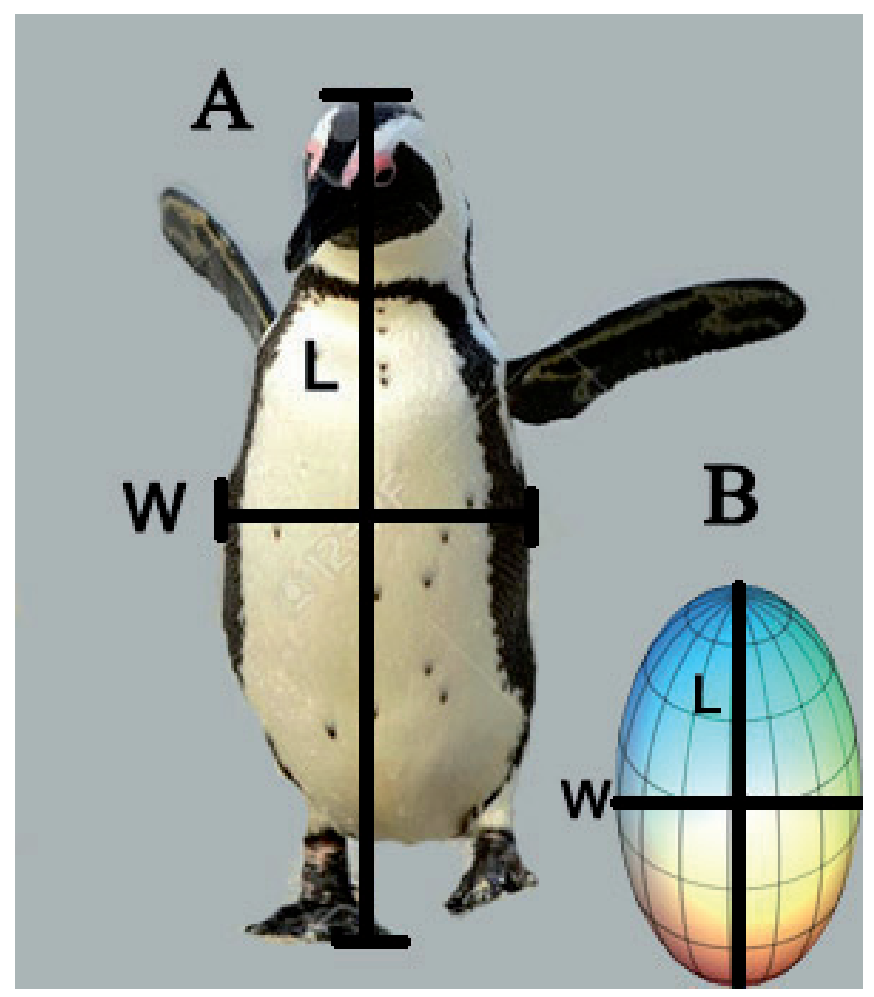

Figura 2. a) Spheniscus demersus. b) esferoide prolato. L: longitud del cuerpo; W: diámetro medio del cuerpo. Modificado de fotografía:

https://www.google.com.pe/search?sa=G\&hl=en-PE\& $\mathrm{q}=$ emperor + penguin $\&$ tbm $=\mathrm{isch} \&$ source $=\mathrm{iu} \& \mathrm{ict} \mathrm{x}=1 \&$ tbs=simg:CAESpgIJ6e0UyVsOcy8amgILELCMpwga YgpgCAMSKMsC6BKGB5kHlgenB4MGhgaABuwS 3SGtLbI39CnyKZQ18ynPKPUp0SgaMOr8MyGe6K NU-R-XxDCINYHdEpbF0Jxc1Eq1q31P7dScDiforIrV KpfNOJDxBvh2NCAEDAsQjq7-CBoKCggIARIEhat-

\subsection{Asunciones y limitaciones}

$\mathrm{Al}$ asumir que la forma del pingüino I. paracasensis es similar a la de $A$. forsteri, se tiene que precisar el concepto de forma para este caso. Desde el punto de vista dinámico, la forma de un pingüino responde a un plan corporal simétrico bilateral fusiforme, con módulos locomotores constituidos por alas y patas palmeadas como timones y propulsores a la vez. 


\subsection{Cálculo de E (excentricidad)}

Inkayacu paracasensis: Reemplazando $\mathrm{L}=150 \mathrm{~cm}$ y $\mathrm{W}=58 \mathrm{~cm}$ en la ecuación (5) obtenemos $\mathrm{E}=0,92$.

Aptenodytes forsteri: Reemplazando $\mathrm{L}=115 \mathrm{~cm}$ y W $=45 \mathrm{~cm}$ en la ecuación (5) obtenemos $\mathrm{E}=0,92$.

Spheniscus demersus: Reemplazando $\mathrm{L}=70 \mathrm{~cm}$ y W $=26 \mathrm{~cm}$ en la ecuación (5) obtenemos $\mathrm{E}=0,93$.

\subsection{Cálculo de A (área del cuerpo)}

Inkayacu paracasensis: Reemplazando $\mathrm{L}=150 \mathrm{~cm}$, $\mathrm{W}=58 \mathrm{~cm}$ y $\mathrm{E}=0,92$ en la ecuación (4) obtenemos $\mathrm{A}=22619 \mathrm{~cm}^{2}\left(2,26 \mathrm{~m}^{2}\right)$. Como ya se explicó en la metodología, este valor asume la forma de Inkayacu como la de un esferoide prolato.

Aptenodytes forsteri: Reemplazando $\mathrm{L}=115 \mathrm{~cm}, \mathrm{~W}$ $=45 \mathrm{~cm}$ y $\mathrm{E}=0,92$ en la ecuación (4) obtenemos $\mathrm{A}=$ $13501,73 \mathrm{~cm}^{2}\left(1,35 \mathrm{~m}^{2}\right)$.

Spheniscus demersus: Reemplazando $\mathrm{L}=70 \mathrm{~cm}, \mathrm{~W}$ $=26 \mathrm{~cm}$ y $\mathrm{E}=0,93$ en la ecuación (4) obtenemos $\mathrm{A}=$ $4733,53 \mathrm{~cm}^{2}\left(0,47 \mathrm{~m}^{2}\right)$.

\subsection{Cálculo de $C_{d}$ (coeficiente de arrastre o resistencia del agua)}

No se considera el $\mathrm{C}_{\mathrm{d}}$ total, que incluye la resistencia o fricción de onda cerca de la superficie, dado que los pingüinos nadan a diferentes profundidades. En el estudio de Kooyman et al. (1992) se registraron individuos de Aptenodytes forsteri nadando a profundidades máximas de 19 a $41 \mathrm{~m}$. Para el componente de fricción de la piel, $\mathrm{C}_{\mathrm{f}}$, se toma el valor de 0,004 (Hoerner, 1965).

Inkayacu paracasensis: Reemplazando $\mathrm{L}=150 \mathrm{~cm}$, $\mathrm{W}=58 \mathrm{~cm} \mathrm{y} \mathrm{C}_{\mathrm{f}}=0,004$ (Hoerner, 1965) en la ecuación (6) obtenemos $\mathrm{C}_{\mathrm{d}}=0,0071$.

Aptenodytes forsteri: Reemplazando $\mathrm{L}=115 \mathrm{~cm}, \mathrm{~W}=$ $45 \mathrm{~cm} \mathrm{y} \mathrm{C}_{\mathrm{f}}=0,004$ en la ecuación (6) obtenemos $\mathrm{C}_{\mathrm{d}}=$ 0,0071 .

Spheniscus demersus: Reemplazando $\mathrm{L}=70 \mathrm{~cm}, \mathrm{~W}=$ $26 \mathrm{~cm}$ y $\mathrm{C}_{\mathrm{f}}=0,004$ en la ecuación (6) obtenemos $\mathrm{C}_{\mathrm{d}}=0,006$.

\subsection{Cálculo de $P$ (potencia total de salida)}

Inkayacu paracasensis: Reemplazando en la ecuación (9) una masa $\mathrm{M}$ estimada de 54,6 a $59,7 \mathrm{~kg}$ (Clarke et al., 2010), con una media de $\mathrm{M}=57,2 \mathrm{~kg}$, y la constante metabólica a de 300 a 530 (Bakker, 1975), entonces obtenemos $\mathrm{P}_{1}=6239,7(\mathrm{a}=300)$ y $\mathrm{P}_{2}=11023,5(\mathrm{a}=530)$.

Aptenodytes forsteri: Reemplazando en la ecuación (9) una masa media $\mathrm{M}=30 \mathrm{~kg}$ (Stonehouse, 1967), y la constante metabólica a de 300 a 530, entonces obtenemos $\mathrm{P}_{1}=3845,6(\mathrm{a}=300)$ y $\mathrm{P}_{2}=6793,9(\mathrm{a}=530)$.
Spheniscus demersus: Reemplazando en la ecuación (9) una masa media $\mathrm{M}=2,9 \mathrm{~kg}$ (Stonehouse, 1967) y la constante metabólica a de 300 a 530, entonces obtenemos $\mathrm{P}_{1}=666,7(\mathrm{a}=300)$ y $\mathrm{P}_{2}=1177,8(\mathrm{a}=530)$.

\subsection{Cálculo de e (proporción de energía disponible para la locomoción)}

Inkayacu paracasensis y Aptenodytes forsteri: Reemplazando en la ecuación (8) $\mathrm{e}_{\mathrm{p}}=0,15$, que es el mínimo entre 0,15 y 0,30 (Blake, 1980), bajo el supuesto de una eficiencia hidrodinámica menor para ambos pingüinos. Esto es debido a que Aptenodytes forsteri tiene una frecuencia máxima de batidos de aleta por segundo de 1,50 en el estudio de Clark \& Bemis (1979), la más baja del grupo muestreado de pingüinos, lo cual implica una menor eficiencia hidrodinámica. Dado que la razón del bajo valor de la frecuencia es solo atribuible a su gran tamaño, se aplicará el mismo criterio a Inkayacu. Además, hemos asumido anteriormente que $\mathrm{e}_{\mathrm{m}}=0,20$ (Massare, 1988). Entonces obtenemos e $=0,03$ para ambos pingüinos.

Spheniscus demersus: $S$. demersus tiene una alta frecuencia máxima de batidos de aleta por segundo, 3,24 , comparada con otras especies de pingüinos, como Aptenodytes forsteri con 1,5 batidos/seg (Clark \& Bemis, 1979). Por lo tanto, su eficiencia hidrodinámica es alta, por eso se tomará el valor máximo de $e_{p}=0,30$. En la ecuación (8) reemplazamos $\mathrm{e}_{\mathrm{p}} \mathrm{y} \mathrm{e}_{\mathrm{m}}=0,20$, entonces obtenemos e $=0,06$.

\subsection{Cálculo de U (velocidad máxima sostenida)}

Inkayacu paracasensis: Reemplazando en la ecuación (10) $e_{p}, e_{m}, P$, densidad del agua de mar $\rho=1025 \mathrm{~kg} / \mathrm{m}^{3}$ (Rivera, 2011), A y $\mathrm{C}_{\mathrm{d}}$, para $\mathrm{P}=6239,7$ obtenemos un valor de $\mathrm{U}=2,8 \mathrm{~m} / \mathrm{seg}$ y para $\mathrm{P}=11023,5$ un valor de $\mathrm{U}=3,4 \mathrm{~m} / \mathrm{seg}$.

Aptenodytes forsteri: Reemplazando en la ecuación (10) $e_{p}, e_{m}, P$, densidad del agua de mar $\rho=1025 \mathrm{~kg} / \mathrm{m}^{3}$, A y $C_{d}$, para $P_{1}=3845,6$ obtenemos un valor de $U=2,8$ $\mathrm{m} / \mathrm{seg}$ y para $\mathrm{P}_{2}=6793,9$ un valor de $\mathrm{U}=3,5 \mathrm{~m} / \mathrm{seg}$.

Spheniscus demersus: Reemplazando en la ecuación (10) $e_{p}, e_{m}, P$, densidad del agua de mar $\rho=1025 \mathrm{~kg} / \mathrm{m}^{3}$, A y $C_{d}$, para $P_{1}=666,7$ obtenemos un valor de $U=3,0 \mathrm{~m} /$ seg y para $P_{2}=1177,8$ un valor de $U=3,7 \mathrm{~m} / \mathrm{seg}$.

\subsection{Modelo de flujo (Re)}

Inkayacu paracasensis: Para $v_{t}$ se toma la media de la velocidad máxima sostenida calculada, $3,1 \mathrm{~m} / \mathrm{seg}$; densidad del agua de $\operatorname{mar} \rho=1025 \mathrm{~kg} / \mathrm{m}^{3}$; longitud estimada $l=1,5 \mathrm{~m}$; y viscosidad dinámica del agua de mar $\mu=1,072 \times 10^{-3} \mathrm{~kg} / \mathrm{ms}$ 
a $20^{\circ} \mathrm{C}$ y $1 \mathrm{~atm}$ de presión (Vogel, 1996). Se reemplaza en la ecuación (11): $\operatorname{Re}=4446129$.

Aptenodytes forsteri: Para $v_{t}$ se toma la velocidad máxima hallada por Kooyman et al. (1992), 3,6 m/seg; longitud estimada $l=1,15 \mathrm{~m}$; la $\rho$ y la son las mismas que en Inkayacu: $\mathrm{Re}=3958489$.

Spheniscus demersus: Para $v_{t}$ se toma la velocidad máxima hallada por Clark \& Bemis (1979), $3,23 \mathrm{~m} / \mathrm{seg}$; longitud estimada $l=0,7 \mathrm{~m}$; la $\rho$ y la son las mismas que en las anteriores: $\mathrm{Re}=2161870$.

\section{DISCUSIÓN}

El método se aplicó a Aptenodytes forsteri y a Spheniscus demersus con el fin de confirmar la eficacia del mismo para pingüinos; esto se evidenció al comparar las velocidades calculadas con las observadas de ambas especies. Clark \& Bemis (1979), utilizando cronómetro y registro en cintas de videos, estudiaron la capacidad de nado de siete especies de pingüinos en cautiverio en el zoológico de Detroit. Para Spheniscus demersus hallaron una velocidad máxima de $3,23 \mathrm{~m} / \mathrm{seg}$, velocidad que se encuentra en el rango hallado analíticamente: 3,0 a 3,7 m/seg. Sin embargo, para Aptenodytes forsteri observaron una velocidad máxima de $2,26 \mathrm{~m} / \mathrm{seg}$, un valor ligeramente menor al mínimo del rango de la velocidad máxima calculada: $2,8 \mathrm{~m} / \mathrm{seg}$, que corresponde a la potencia total de salida $(\mathrm{P})$, medida como la tasa metabólica aeróbica máxima (Massare, 1988), con una constante metabólica a igual a 300, que es el valor mínimo de la energía máxima a través de la actividad metabólica aeróbica para mamíferos y aves (convertido de la energía máxima a través del metabolismo del ejercicio aeróbico en Bakker, 1975: Tabla 21.1). Sin embargo, Kooyman et al. (1992) en un laboratorio con hielo en el mar y colocando microprocesadores en el cuerpo del animal que, entre otras variables, mide velocidad, observaron en nado de buceo a profundidad de A. forsteri un rango de 2,4 y $3,4 \mathrm{~m} / \mathrm{seg}(\mathrm{N}=4)$, con una media de $2,8 \mathrm{~m} / \mathrm{seg}$. Este resultado es solo referencial, ya que el método de Massare (1988) no considera nado de buceo a profundidad. Sin embargo, el mismo trabajo (Kooyman et al., 1992; Tabla 5) registra velocidades de nado obtenidas de 5.681 mediciones a ocho pingüinos $A$. forsteri, que arrojan una velocidad media de $2,98 \mathrm{~m} / \mathrm{seg}$, observándose en el análisis de distribución de frecuencias que las velocidades más comunes están entre 3 y $4 \mathrm{~m} / \mathrm{seg}$, y la velocidad de calibración máxima de 3,6 m/seg.

En este mismo estudio se registraron velocidades máximas en un rango de 4,6 a 7,1 m/seg. Sin embargo, de estos resultados máximos la investigación de Kooyman et al. (1992) no presenta una media de velocidad máxima porque son cifras que están en el extremo del eje de velocidad, de los valores máximos, en la distribución de frecuencias, cuya ocurrencia es baja. Así mismo, son pocos los casos de velocidades bajas. Estos valores máximos no serán considerados para el presente trabajo.

Kooyman et al. (1992) también hallaron algunas velocidades de nado directamente de la cámara de observación, donde la tasa media de $2.5 \pm 0.86 \mathrm{~m} / \mathrm{seg}(\mathrm{N}=$ 13) es menor que la medida con los medidores de velocidad de los microprocesadores. Según los investigadores, lo reducido de la muestra y el descenso de las aves en esta medición probablemente marquen la diferencia con las mediciones con microprocesador. Aquí, dicho estudio menciona una velocidad máxima observada de $3,8 \mathrm{~m} / \mathrm{seg}$.

La media de la velocidad máxima calculada de Aptenodytes forsteri es 3,2 m/seg. Este valor es comparado con las velocidades máximas observadas: $2,26 \mathrm{~m} / \mathrm{seg}$ (Clark \& Bemis, 1979) y $3,6 \mathrm{~m} / \mathrm{seg}$ (con microprocesadores en Kooyman et al., 1992). Las discrepancias en los valores de ambos trabajos son sugeridos por Clark \& Bemis (1979), en una limitación de la capacidad de crucero de los pingüinos, aunque no severa, del pingüinario de Detroit. Hay que tener en cuenta que ni en el estudio de ellos ni en el de Kooyman et al. (1992) fueron los animales obligados a nadar a su máxima capacidad.

Promediando los valores de Clark \& Bemis (1979) y Kooyman et al. (1992) obtenemos $2,9 \mathrm{~m} / \mathrm{seg}$, es decir, el valor calculado difiere en $+0,3 \mathrm{~m} / \mathrm{seg}$ que equivale al $10,3 \%$ con respecto a la media del valor observado máximo de ambos trabajos. Entonces, se puede concluir que los resultados calculados son bastante cercanos a los observados. Podemos afirmar, por lo tanto, que el modelo es una buena aproximación a las velocidades reales, afirmación que se sustenta con las dos especies de pingüinos actuales. En consecuencia, por los resultados obtenidos del pingüino Emperador y de Inkayacu, ambas especies habrían nadado a una velocidad muy cercana.

El nado del pingüino, aparte de la velocidad, tiene otras características, como el ciclo de batido de la aleta, la frecuencia del batido de la aleta, la amplitud del batido de la aleta, la longitud de la zancada, las fases de deslizamiento y el control direccional (Clark \& Bemis, 1979). En este trabajo se han abordado las características que tienen relación directa con la velocidad: la frecuencia del batido de la aleta, la longitud de la zancada y las fases de deslizamiento. Estos investigadores afirman que para la mayoría de pingüinos la frecuencia y la longitud de la zancada son moduladas con la velocidad, aunque esta relación puede ser menos pronunciada o ausente cuando los pingüinos utilizan fases de deslizamiento, equivalente al planeo de las aves voladoras. En términos físicos, esta última característica implica, debido a la resistencia del agua, una desaceleración o pequeños tramos de velocidad casi constante.

Los pingüinos baten sus aletas continuamente al nadar a velocidad constante de forma horizontal. Sin embargo, todas las especies interponen fases de deslizamiento, sin 
potencia entre batidos, sobre todo cuando la velocidad de nado es menor de $1,25 \mathrm{~m} / \mathrm{seg}$ (Clark \& Bemis, 1979). Los pingüinos que típicamente interponen fases de deslizamiento entre golpes o batidos de aleta en un rango más amplio de velocidades que otras especies son el pingüino Emperador, King y Adelie (Clark \& Bemis, 1979), que son los más grandes del estudio. Estos investigadores también han observado que las especies que muestran más fases de deslizamiento son las de tamaño corporal más grande porque el arrastre (resistencia del agua) en un cuerpo aerodinámico es una función de su área de superficie, mientras que el impulso o momentum es una función de su masa, expresado en la fórmula $\mathrm{P}=$ $\mathrm{m} v$, donde $\mathrm{m}$ es la masa y $v$ la velocidad del cuerpo. Por lo tanto, los pingüinos más grandes de largos deslizamientos o planeadas desaceleran más lentamente que los pequeños, es decir, por su masa pueden mantener el nado sin batido de aletas por más tiempo. Entonces, dada la gran masa, forma y velocidad semejantes, Inkayacu habría tenido un nado con las características de fases de los pingüinos más grandes actuales. Además, siendo más específicos, se puede afirmar que Inkayacu tenía fases de deslizamiento más largas y de mayor duración que las del pingüino Emperador debido a que su masa es casi el doble que la del ave actual, con medias de $57,2 \mathrm{~kg}$ contra $30 \mathrm{~kg}$. Esta afirmación está sustentada en lo que dice Eckert et al. (1994), que la fuerza es directamente proporcional a la masa muscular y, asumiendo que la masa muscular aumenta en proporción a la masa corporal total, la fuerza aumenta en proporción a esta masa corporal. Además, para un animal nadador grande a una velocidad determinada, la resistencia al avance por unidad de masa disminuye con la masa del cuerpo. Por tanto, el animal acuático más grande, con respecto al animal más pequeño, puede desarrollar una fuerza mucho mayor en proporción a la fuerza que produce la resistencia al avance.

Según Wardle (1975), la longitud de la zancada es la distancia recorrida por aleteo y se mide en longitudes de cuerpo. La velocidad también se puede medir en longitudes de cuerpo/seg, tal como lo hace Clark \& Bemis (1979), observándose que en las especies más pequeñas de pingüinos la velocidad aumenta con la longitud de la zancada, mientras que en los más grandes, como el pingüino Emperador, la velocidad es relativamente constante en un amplio rango de longitudes de zancada. En la característica de nado longitud de la zancada, se puede afirmar que, por su gran tamaño, Inkayacu habría mantenido una velocidad relativamente constante en un rango amplio de longitudes de zancada porque, al igual que las fases de deslizamiento, depende principalmente de parámetros físicos.

En cuanto al buceo, Kooyman et al. (1971) sugieren que la frecuencia del aleteo y la longitud de la zancada muestran poca o ninguna correlación con la velocidad en el buceo del pingüino Emperador. En nado horizontal para el pingüino Emperador, Kooyman et al. (1971) observaron una frecuencia de batido de aletas de 0,4 a 0,8 batidos/ seg, mientras que para Clark \& Bemis (1979) fue de 1 a 2 batidos/seg. Sin embargo, los rangos de batidos por segundo son equivalentes cuando se toman en cuenta las fases de deslizamiento, que ambos estudios han incluido.

Clark \& Bemis (1979) comparan el nado de los pingüinos con el de otros grupos de animales y han encontrado que se asemeja más al nado de los peces, en particular a la locomoción de la perca Cymatogaster aggregata, que al vuelo de las aves. Solo los pingüinos y Cymatogaster aumentan la frecuencia y la longitud de la zancada con la velocidad. Según Breder (1926), la similitud de la cinemática de la locomoción de los pingüinos y Cymatogaster puede ser el resultado de modos de locomoción similares, ya que ambos tienen un nado labriforme. Dado que Clark \& Bemis (1979) han demostrado que los pingüinos generalmente modulan la velocidad con la frecuencia del batido de la aleta y la longitud de la zancada. Es decir, generalmente hay una relación directamente proporcional entre la velocidad de nado horizontal y la frecuencia de batido de aleta. Además, el pingüino Emperador tiene una frecuencia máxima de batidos de aleta por segundo menor que la mayoría de pingüinos, lo que justifica nuevamente relacionar el tamaño del animal con el desempeño de esta característica de nado y afirmar, por ende, que Inkayacu habría tenido una frecuencia de batidos de aleta por segundo baja también (de ahí la suposición en Resultados de una eficiencia hidrodinámica menor para ambos pingüinos) y una velocidad de nado similar a Aptenodytes forsteri, como ya se ha demostrado teóricamente por el método biomecánico.

Otro factor que influye en la velocidad de un animal nadador es el modelo de flujo, que el método de Massare (1988) no considera. El análisis del modelo de flujo que se hace a continuación, no se infiere del modelo de la investigadora, el cual tiene sus propias limitaciones y condiciones específicas sin considerar modelo de flujo. A un bajo $\operatorname{Re}(\operatorname{Re}<1)$ las fuerzas viscosas son más importantes que las inerciales (Jou et al., 1994) y, además, se produce un modelo de flujo puramente laminar al pasar el líquido por su superficie; es el caso de una pequeña larva. Un rango siguiente es el intermedio, no bien definido, donde la viscosidad y la inercia son importantes, como sucede con el vuelo de un mosquito. A Re mayores que 40 empieza a aparecer turbulencia en la estela del cuerpo en movimiento (Eckert et al., 1994). En movimientos a altos Re la inercia es el factor importante (Jou et al., 1994), y si Re es mayor que 1.000 .000 el fluido en contacto con la superficie del cuerpo se hace turbulento. En este nivel, la energía necesaria para un mayor incremento de la velocidad aumenta desorbitantemente (Eckert et al., 1994). Los Re obtenidos de los pingüinos, para Inkayacu, $\operatorname{Re}=4.446 .127$; Emperador (A. forsteri), $\operatorname{Re}=3.958 .489$; y Spheniscus demersus, $\mathrm{Re}=2.161 .870$, nos muestran que 
en los tres casos el fluido se hace turbulento en contacto con el cuerpo. Este modelo de flujo es más pronunciado en los pingüinos más grandes, en particular en Inkayacu, cuyo Re es el $112,33 \%$ del Re de $A$. forsteri, por lo que para nadar a mayor velocidad Inkayacu necesita gastar un poco más de energía que los otros pingüinos. Aunque el 12,33\% de diferencia con el pingüino Emperador (en números de Reynolds igual a 487.638), es poco significativo en términos de valores de modelos de flujo, porque ambos son turbulentos. Los Re obtenidos no consideran la forma de los pingüinos. Al respecto, Hoerner (1965) sugiere que la forma aerodinámica del cuerpo de los pingüinos resulta en una presión muy pequeña de arrastre (resistencia del agua) y que la capa límite sobre los pingüinos Emperador deslizándose es en parte laminar y en parte turbulento. Esta condición de transición es típica en el rango de números de Reynolds de alrededor de $10^{6}$.
Un caracter de su morfología que favorece a Inkayacu y compensa la mayor turbulencia generada es la forma del cráneo. Como se aprecia en la Figura 3 y la Figura 4, a diferencia de los pingüinos actuales y de varias especies extintas, el cráneo de Inkayacu es largo y estilizado, más aerodinámico que el cráneo del pingüino Emperador. Sobre la forma aerodinámica de los animales, en general, Eckert et al. (1994) afirma que las formas largas y aerodinámicas facilitan el flujo laminar, minimizando la formación de corrientes en remolino, como los peces y cetáceos, que consiguen pasar a través del agua casi sin turbulencia incluso a elevadas velocidades. Esta es una ventaja no considerada en los cálculos, que Inkayacu tiene y que influiría en una ligera mayor velocidad. En este caso, la aparente igualdad de velocidades entre Inkayacu y el pingüino Emperador se rompería, pero no sabemos por cuánto.

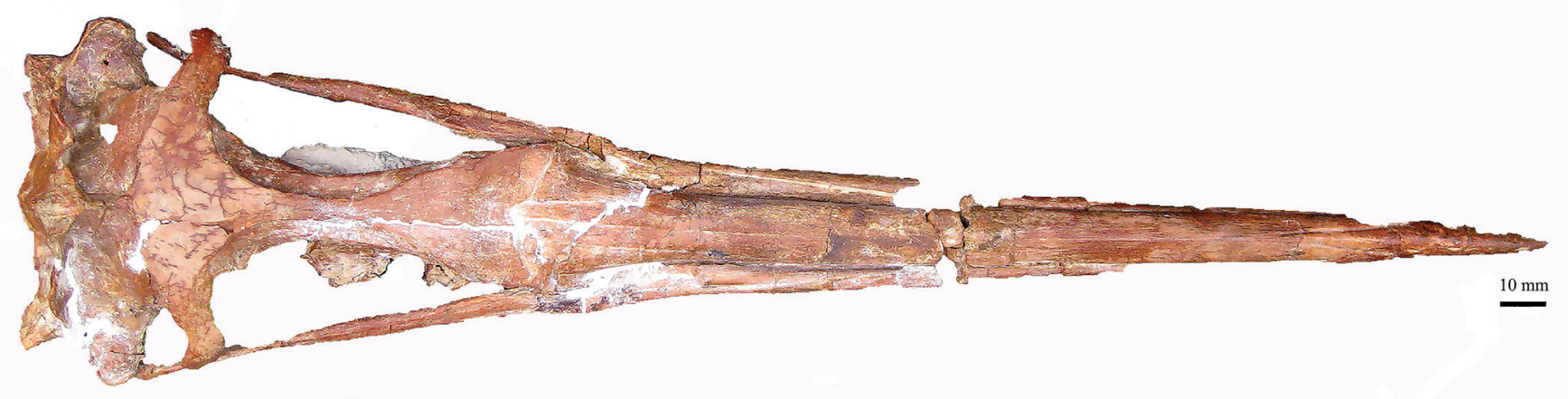

Figura 3. Holotipo de Inkayacu paracasensis (MUSM 1444). Cráneo en vista dorsal (Departamento de Paleontología de Vertebrados del Museo de Historia Natural-UNMSM). Modificación de fotografía de Rodolfo Salas Gismondi.

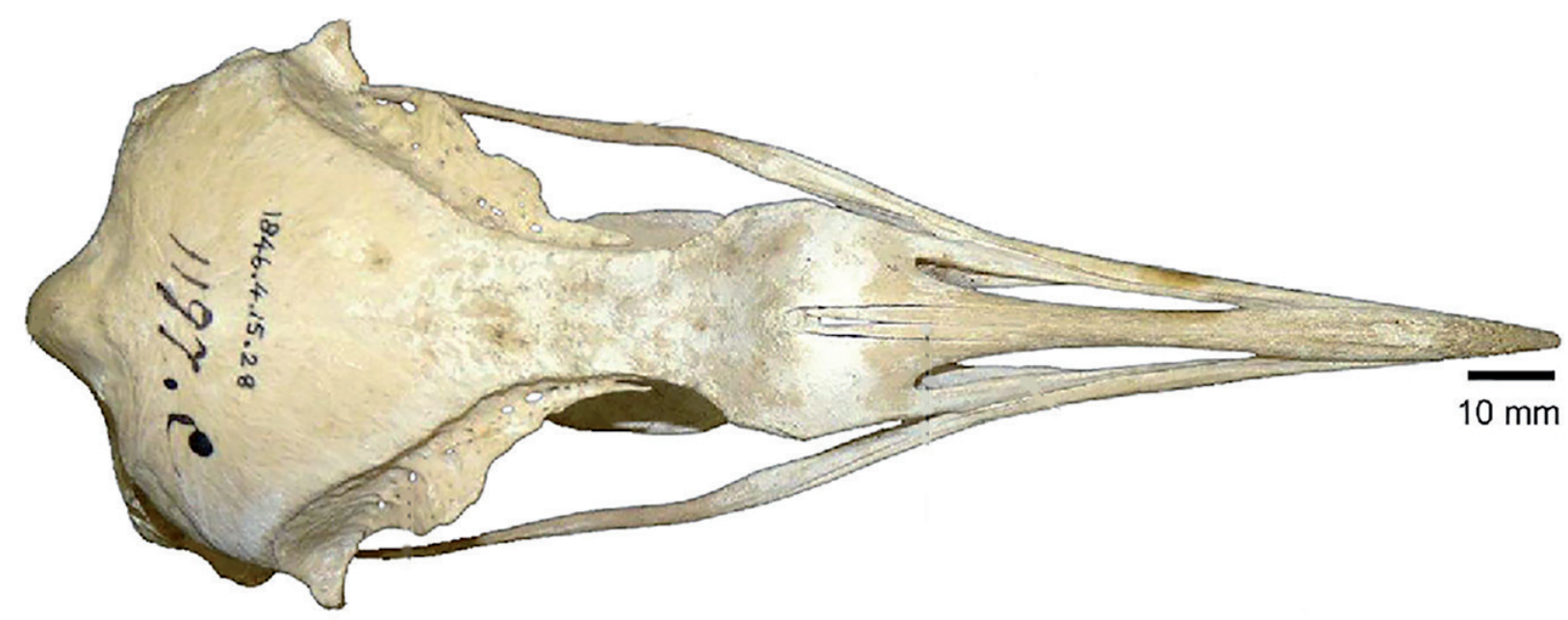

Figura 4. Cráneo de un adulto de Aptenodytes forsteri en vista dorsal. Modificación de fotografía de María Alejandra Sosa (Sosa \& Acosta, 2017). 
El presente trabajo solo considera un aspecto del rendimiento o capacidad del nado, la velocidad, pero la aceleración y la resistencia del animal también forman parte, y los tres están directamente relacionados con la capacidad para usar con eficiencia los recursos energéticos disponibles en respuesta a los requerimientos hidrodinámicos en el balance entre el empuje generado por el animal nadador y el arrastre o resistencia del agua (Weihs \& Webb, 1983). Otro aspecto que no se considera son las implicancias ecológicas de este estudio, como en los métodos de depredación, etc. Por lo tanto, este trabajo es el inicio de un mayor estudio sobre la capacidad de nado y sus implicancias en los pingüinos fósiles.

\section{AGRADECIMIENTOS}

A mis amigos del Departamento de Paleontología de Vertebrados del Museo de Historia Natural de la UNMSM, Rafael Varas Malca por la edición del inglés del resumen y a Aldo Benites Palomino por la asesoría en la edición de fotografía.

\section{REFERENCIAS}

Alexander, R.M. 1968. Animal Mechanics. University of Washington Press, Seattle.

Bainbridge, R. 1961. Problems of fish locomotion. Zoological Society of London, Symposia, 5, 13-32.

Bakker, R.T. 1975. Experimental and fossil evidence for the evolution of tetrapod bioenergetics. In: Ecological Studies, Analysis and Synthesis, Volume 12 (eds Gates, D.M. \& Schmerl, R.B.). Perspectives of Biophysical Ecology Symposium. Ann Arbor, Michigan. Springer-Verlag, New York. 365-399.

Blake, R.W. 1980. The mechanics of labriform locomotion II. An analysis of the recovery stroke and the overall finbeat cycle propulsive efficiency in the angelfish. Journal of Experimental Biology, 85, 337-342.

Breder, C. 1926. The locomotion of fishes. Zoologica, 4, 159-297.

Clark, B. \& Bemis, W. 1979. Kinematics of swimming of penguins at the Detroit Zoo. Journal of Zoology, 188, 411-428.

Clarke, J., Ksepka, D., Salas-Gismondi, R., Altamirano, A., Shawkey, M., Alba, L., Vinther, J., DeVries, T. \& Baby, P. 2010. Fossil evidence for evolution of the shape and color of penguin feathers. Sciencexpress, 3, 1-4; doi: 10.1126/ science. 1193604.

Eckert, R., Randall, D. \& Augustine, G. 1994. Fisiología Animal. $3^{a}$ Edición. Interamericana-McGraw-Hill.

Gray, G.R. 1844. Aptenodytes. Annals and Magazine for Natural History, 13, 315.
Hatze, H. 1974. The meaning of the term: Biomechanics. Journal of Biomechanics, 7, 189-190.

Hoerner, S.F. 1965. Fluid Dynamic Drag. Brick Town, New Jersey. Published by the author.

Jou, D., Llebot, J. \& Perez, C. 1994. Física para Ciencias de la Vida. McGraw-Hill. 526 pp.

Kleiber, M. 1947. Body size and metabolic rate. Physiological Review, 27, 511-541.

Kooyman, G.L., Ponganis, P.J., Castellini, M.A., Ponganis, E.P., Ponganis, K.V., Thorson, P.H., Eckert, S.A. \& LeMaho, L. 1992. Heart rates and swim speeds of emperor penguins diving under sea ice. Journal of Experimental Biology, 165, 161-80.

Linnaeus, C. 1758. Systema Naturae per Regna Tria Naturae, Secundum Classes, Ordines, Genera, Species, cum Characteribus, Differentiis, Synonymis, Locis. Laurentii Salvii, Holmiae. Vol. Tomus I, Editio decima, reformata: i-ii, $1-824$.

Livezey, B.C. 1989. Morphometric patterns in recent and fossil penguins (Aves, Sphenisciformes). Journal of Zoology, 219, 269-307.

Luna-Jorquera, G. \& Culik, B. 2000. Metabolic rates of swimming Humboldt penguins. Marine Ecology Progress Series, 203, 301-309.

Massare, J. 1988. Swimming capabilities of Mesozoic marine reptiles: implications for method of predation. Paleobiology, 14, 187-205; doi: 10.1017/S009483730001191X.

Raikow, R.J. 1985. Locomotor system. In: Form and Function in Birds (eds King, A.S. \& McLelland, J.). London, Orlando. Academic Press, 3, 57-147.

Rivera, H. 2011. Geología General. Grupo Editorial Megabyte, Lima.

Shockey, B., Croft, D. \& Anaya, F. 2007. Analysis of function in the absence of extant functional homologues: a case study using mesotheriid notoungulates (Mammalia). Palaeontology, 33, 227-247; doi: https:// doi.org/10.1666/05052.1.

Simpson, G.G. 1946. Fossil penguins. Bulletin of the American Museum of Natural History, 87, 1-99.

Slack, K., Jones, C., Ando, T., Harrison, G., Fordyce, R., Arnason, U. \& Penny, D. 2006. Early penguin fossils, plus mitochondrial genomes, calibrate avian evolution. Molecular Biology and Evolution, 23, 1144-1155; doi: 10.1093/molbev/msj124.

Sosa, M. \& Acosta, C. 2017. Ontogenetic variations of the head of Aptenodytes forsteri (Aves, Sphenisciformes): muscular and skull morphology. Polar Biology, 41, 225-235; doi: 10.1007/s00300-017-2183-3.

Stonehouse, B. 1967. The general biology and thermal balances of penguins. Advances in Ecological Research, 4, 131-196.

Stonehouse, B. 1975. Introduction: the Spheniscidae. In: The Biology of Penguins (ed. Stonehouse, B.). University Park Press, London. 1-15.

Vogel, S. 1996. Life in Moving Fluids: the Physical Biology of Flow. $2^{a}$ Edition. Princeton University Press, Princeton.

Wardle, C. 1975. Limit of fish swimming speed. Nature, 255, 725-727. 
Webb, P.W. 1977. Effects of fish size on performance and energetics of fish. In: Scale Effects in Animal Locomotion (ed. Pedley, T.J.). Academic Press, New York. 315-331.

Weihs, D. 1977. Effects of size on sustained swimming speeds of aquatic organisms. In: Scale Effects in Animal Locomotion (ed. Pedley, T.J.). Academic Press, New York. 333-338.

Weihs, D. \& Webb, P. 1983. Optimization of locomotion. In: Fish Biomechanics (eds Webb, P.W. \& Weihs, D.). Praeger, New York. 339-371.

Wilkie, D.R. 1977. Metabolism and body size. In: Scale Effects in Animal Locomotion (ed. Pedley, T.J.). Academic Press, New York. 23-36.
Williams, T. 1995. The Penguins, Spheniscidae. Oxford University Press.

$\mathrm{Wu}, \mathrm{T}$. Y. 1977. Introduction to the scaling of aquatic animal locomotion. In: Scale Effects in Animal Locomotion (ed. Pedley, T.J.). Academic Press, New York. 203-232.

Yavorski, B. \& Detlaf, A. 1977. Manual de Física. 2nd Edition. Mir. Moscú.

Zusi, R. 1975. An interpretation of skull structure in penguins. In: The Biology of Penguins (ed. Stonehouse, B.). University Park Press, London. 59-84. 
